\title{
El método de construcción del relato fílmico y el estilo cinematográfico de Mike Leigh: reflejo de una concepción ética y estética del mundo
}

\author{
Mike Leighen zinemagintza-estiloa eta filmetako \\ kontakizuna eraikitzeko metodoa: munduaren \\ ikusmolde etiko eta estetiko baten isla
}

\section{Mike Leigh's method to build up a filmic narrative and cinema style: reflection of an ethic and aesthethic conception of the world}

\section{Laura Cortés-Selva ${ }^{1}$ \\ María Mercedes Carmona-Martínez ${ }^{2}$}

\section{zer}

Vol. 21 - Núm. 40

ISSN: $1137-1102$

e-ISSN: $1989-631 \mathrm{X}$

DOI: $10.1387 /$ zer. 15513

pp. 109-123

2016

Recibido el 30 de junio de 2014, aceptado el 14 de diciembre de 2015.

\begin{abstract}
Resumen
La concepción ética y estética del director de cine Mike Leigh impregna de tal manera su obra cinematográfica que sus filmes se diferencian de los englobados en el cine comercial norteamericano. El objetivo principal de esta investigación se centra en analizar su método, así como algunos de los elementos más importantes que configuran su estilo, y en qué se diferencia del cine comercial norteamericano. Se ha combinado una metodología cualitativa y cuantitativa que hibrida la consulta de las principales fuentes que versan sobre el autor, así como el análisis cuantitativo de aquellos elementos más relevantes de su estilo cinematográfico.
\end{abstract}

Palabras clave: Mike Leigh, guión, actores, método, estilo cinematográfico.

\footnotetext{
Universidad Católica de Murcia, LCortes@ucam.edu

2 Universidad Católica de Murcia, Mcarmona@ucam.edu
} 


\title{
Laburpena
}

Mike Leigh zinema-zuzendariaren ikuspegi etiko eta estetikoak goitik behera bustitzen ditu haren zinema-lanak; hori dela eta, haren filmak ez dira Ipar Amerikako zinema komertzialaren etiketapean sailkatzen direnak bezalakoak. Ikerketa honen helburu nagusia da haren metodoa aztertzea, bai eta haren estiloa osatzen duten elementu garrantzitsuenetako batzuk aztertzea ere, Ipar Amerikako zinema komertzialetik bereizten duten ezaugarriak ahaztu gabe. Horretarako, metodologia kualitatiboa eta kuantitatiboa konbinatu dira: alde batetik, autoreari buruzko iturri nagusiak kontsultatu dira eta, bestetik, haren zinema-estiloaren elementurik garrantzitsuenak kuantitatiboki aztertu dira.

Gako-hitzak: Mike Leigh, gidoia, aktoreak, metodoa, zinema-estiloa.

\begin{abstract}
British Mike Leigh's ethic and aesthetic conception of the world influences his films in a way that they become different from those included in northamerican mainstream cinema, specially in his method and style. The main objective of this research is focused upon analyzing Leigh's mentioned conception through his method and some of the most important elements conforming his cinema style. To that end, a qualitative and a quantitative methodology that mixes the analysis of the main bibliographical sources about Mike Leigh and a quantitative anaysis of the most important elements conforming his cinema style of his more international acclaimed films, has been conducted.
\end{abstract}

Keywords: Mike Leigh, script, actors, method, cinema style. 


\section{Introducción}

Mike Leigh es uno de los directores de cine británico más importantes e iconoclastas a nivel internacional. No obstante, aunque activo desde principios de los años setenta, su obra despierta la atención de la audiencia internacional a partir de los años noventa, coincidiendo con el máximo galardón que recibe en Cannes por el filme Naked.

La mayoría de las obras que versan sobre el autor están focalizadas en su biografía o en la descripción de su obra cinematográfica (Whitehead, 2007; Raphael, 2008; O’Sullivan, 2011; Movshovitz, 2000; Coveney, 1996), así como las dedicadas a la traducción del guión de sus películas (Leigh, 2002, 1999, 1997a, 1997b y 1995). Existe -por lo tanto- una reducida reflexión académica en torno a la figura del autor británico (Carney, 2000; Watson, 2004), así como escasas publicaciones que se centren en el análisis de su obra. Sin embargo, el director de cine posee un método de trabajo inusual (Clements, 1983) que aplica para la construcción de sus relatos fílmicos, que ha servido de inspiración tanto a investigadores (Irvine, 2008) como a directores.

El objetivo principal de esta investigación se centra en descubrir cuál es la filosofía de Leigh, cuáles son los presupuestos éticos y estéticos más relevantes del universo del creador y cómo se traducen en sus filmes para que se diferencien de los pertenecientes al cine comercial norteamericano. Para ello, se investiga en qué consiste el método que emplea para la construcción de su obra fílmica y los elementos más relevantes configuradores de su estilo cinematográfico.

El objeto de estudio de esta investigación se centra en los filmes de Mike Leigh más conocidos a nivel internacional: Life is sweet (1990), Naked (1993), Secrets and lies (1996), Career girls, 1996), Topsy-Turvy, 1999), All or nothing, 2002), Vera Drake, 2004) y Happy-go-Lucky (2008).

La reflexión propuesta en esta investigación se estructura en torno a tres ejes fundamentales: los antecedentes del método de trabajo de Mike Leigh, el método de trabajo propiamente dicho y la descripción de las características más importantes que definen los elementos más relevantes configuradores del estilo cinematográfico del autor.

\section{Metodología}

Con la intención de demostrar cómo el cine de Mike Leigh posee una firme voluntad por alcanzar la verdad, que él contrapone con lo imperante en el cine comercial norteamericano, es necesario entender su concepción ética y estética.

De ahí la necesidad de analizar los dos ámbitos más importantes que definen la labor de un director de cine: su método de trabajo con los actores y su estilo cinematográfico puesto de manifiesto a través de los elementos configuradores de la puesta en escena.

El método empleado para abordar ambos campos es diferente. Para analizar el método actoral de Leigh se ha optado por una investigación histórica basada en la revisión de las principales fuentes que versan sobre el autor y su obra, y que se detallan en la introducción de este artículo.

Para abordar el análisis del estilo del autor se ha recurrido a la hibridación de técnicas cuantitativas y cualitativas que el lector encontrará detalladas en Cortés-Selva 
y Carmona-Martínez (2014: 819-824 y 832) y en Cortés-Selva (2013: 493), con la intención de obtener una visión lo más completa posible de la temática analizada. En concreto, los elementos estilísticos que se van a analizar son: la tipología y la duración de los planos y los movimientos de cámara (Cortés-Selva y Carmona-Martínez, 2014: 821-825 y 830).

La duración del plano en segundos es una variable que ofrece información sobre la longitud de cada plano individual, lo que nos indica también un estilo de puesta en escena en profundidad o basada en el montaje.

La media de la duración de la totalidad de los planos advierte sobre un acercamiento estilístico más o menos alejado del cine comercial norteamericano, que entre 1988-1993 se caracteriza por una media de 5,85 segundos, que desciende a 4,92 segundos entre 1994-1999. Por el contrario, podría estar más próxima a la del cine británico, que entre 1988-1993 posee una media de 7,4 segundos, que se reduce a 6,8 segundos entre 1994-1999 (Salt, 2006: 321-325).

La duración del plano también anuncia la cantidad de tiempo que ocupa la información que se visualiza y por lo tanto, el peso de la misma en la narración.

La escala del plano es una variable que alude a las dimensiones (altura y anchura) del encuadre, que determinan no sólo la cercanía o lejanía de los personajes a la cámara, sino la composición del encuadre.

Los movimientos de cámara nos hablan de una concepción estética del mundo puesto que existen directores cuya cámara no se desplaza a menos que sea estrictamente necesario en términos narrativos. En la realidad, la cámara, el punto de vista del espectador, no se mueve, sino que permanece quieto, observando.

Aunque se es consciente de que existen otros muchos aspectos susceptibles de análisis y que configuran el estilo del autor (relacionados con la banda sonora, con el montaje, con la dirección artística y con la fotografía) se ha optado por estos tres elementos basándonos en las categorías que definen lo que Bordwell (2006: 121-122) denomina continuidad intensificada (intensified continuity), que -según el autor- caracteriza al cine comercial norteamericano desde la década de los ochenta hasta la actualidad.

\section{El método de trabajo de Mike Leigh}

En este apartado se ofrecen los resultados obtenidos tras la aplicación de una metodología cualitativa basada en el análisis pormenorizado del método de trabajo de Mike Leigh a través de la revisión de las principales fuentes bibliográficas que versan sobre el autor y su obra.

\subsection{Los orígenes}

Las raíces del método de trabajo de Mike Leigh se encuentran en la comedia británica (radiofónica, televisiva y cinematográfica), en el teatro, el vaudeville y el circo, así como en las películas de los hermanos Marx, Laurel y Hardy, Chaplin y Keaton.

Su traslado a Londres en 1960 es fundamental para su formación multidisciplinar en el ámbito de la creación artística. Allí descubre el cine internacional de la mano de Eisenstein, Fellini, Bergman, Satyajit Ray, Buñuel, Ozu o Kurosawa. Directores 
de cine francés como Renoir, Clair o Vigo, le influencian de forma especial, así como los filmes A bout de souffle, (Jean Luc Godard, 1960), Les quatre cents coups (François Truffaut, 1959) o Jules et Jim (François Truffaut, 1961). Shadows (John Cassavetes, 1959) es un filme que también le produce una profunda impresión, específicamente en lo concerniente al resultado en la pantalla del trabajo de improvisación con los actores.

Su formación como actor en la Royal Academy of Dramatic Arts (RADA) no es especialmente significativa para el desarrollo de su método de trabajo actoral, ya que -según Leigh- no se fomentaba la investigación sobre la vida real, sino que repetían esquemas preconcebidos. Por el contrario, sí se promueve la investigación en la escuela de arte a la que Leigh acude (Camberwell Art School), y donde descubre que puede existir un paralelismo entre el dibujo al natural y los ensayos con los actores en cuanto al descubrimiento de algo real y significativo para cada artista (Raphael, 2008: 17).

Participa como actor, durante un periodo de tiempo corto, en la Royal Shakespeare Company, compañía en la que su máximo exponente -Peter Brook- supone una gran influencia para el director británico. Leigh profundiza en su trabajo experimental que le conduce hasta Grotowski y Artaud, y más tarde a Marat/Sade (Marat/Sade, Peter Brook, 1967). El trabajo de Grotowski lo lleva a penetrar más profundamente en el mundo interior del actor, hasta el punto en que este deja de ser actor para convertirse en personaje (Raphael, 2008: 12).

En 1965, cuando se convierte en un director asociado del centro de arte de Birmingham (Midlands Art Centre), comienza a trabajar su método con actores adolescentes. Al lanzar su carrera como director de cine a principios de los años setenta, también asiste a la escuela de cine de Londres, y tras refinar su método en diferentes seminarios y a través del teatro experimental, adopta un estilo de dirección de actores característico que le ha acompañado durante su carrera (Movshovitz, 2000: 47-48).

Con más de cuarenta obras tanto para el teatro como para la gran pantalla, su trabajo para la BBC durante una década ofrece el fruto de películas como Nuts in May (1974), Abigail's party (1977) o Four days in July (1985).

Con el productor Simon Channing-Williams crea Thin Man Films, productora en la que realiza su primer filme -Life is sweet-con la que inaugura la etapa en la que le llega el reconocimiento internacional que comienza con la palma de oro en Cannes -en 1993- por Naked. No obstante, el mayor éxito de taquilla de Leigh -hasta la fecha- es Secrets and lies, galardonado en 1996 con la palma de oro en Cannes y nominado por la academia de cine de Hollywood.

Otra de las obras que recibe un reconocimiento internacional es Vera Drake (2004). Tanto Leigh como su actriz protagonista - Imelda Stauton- son galardonados en el festival de Venecia y nominados a los premios de la Academia de Hollywood.

\subsection{El método propiamente dicho: un viaje hacia el descubrimientode un filme}

Come and be in my film. Can't tell you what it's about. I can't tell you what your character is. We'll invent that as part of the process. And you will never know any more than your character knows. (Cardullo, 2010: 12) 
Comprometido con hacer películas sobre el mundo real tal y como lo conocemos, a Mike Leigh le preocupan cuestiones que afectan a las vidas de la gente común. Gran defensor del sentido tragicómico de la vida, sus filmes reflexionan acerca del paso del tiempo, la clase social, la familia o la ideología.

Leigh ha ido perfeccionando un método para la creación de sus películas que define como orgánico. Se trata de un proceso muy colaborativo en el que comparte con todo el equipo -especialmente con los actores- los aspectos del trabajo final. Aunque Leigh presume de que no existe un autor muy claro en sus filmes, es él quien decide finalmente sobre lo que va a suceder en la narración, los personajes, sus relaciones, etc. (O’Sullivan, 2011: 150).

Como eje central del método de Leigh se encuentran los actores y entre las preocupaciones más importantes del director destaca el hecho de que la audiencia piense que están actuando. Distingue entre las personas reales y las que aparecen en las películas y atribuye la naturalidad presente en sus filmes a no tener que reducir todo a una fórmula, incluso antes de empezar a ser creativo. La fuerza del cine de Leigh consiste en exhibir a los personajes en la pantalla como la gente real, tal y como es, única e individual. De ahí que las caracterizaciones de sus personajes incluyan defectos físicos y de comportamiento que normalmente son eliminados de los personajes que aparecen en la mayoría de las películas. De hecho, uno de los ejes centrales del método de Mike Leigh es su aspiración a la condición de documentalista. Piensa que todo director de cine debería transmitir la sensación de que lo que está narrando audiovisualmente está sucediendo de verdad, con existencia independiente de su filmación. No obstante, todo director de cine no debería dejar de lado la parte teatral, puesto que ningún trabajo artístico es totalmente naturalista, el arte no es la vida real y ha de ser organizado, estructurado para ser dramático (Movshovitz, 2000: 27 y 132).

Para el director, la mayoría de los filmes -especialmente los americanos- están cargados de estereotipos y generalizaciones, tienen sus puntos de giro y momentos climáticos. En el cine de Leigh no siempre existe una estructura clásica y los personajes no son ni héroes ni villanos. Véase por ejemplo el personaje de Johnny -David Thewlis- en Naked o la protagonista de Vera Drake.

Capaces de pensar en profundidad acerca de sus personajes y al mismo tiempo de responder emocionalmente, Leigh trabaja con actores de gran versatilidad, inteligencia y capacidad. Muchos de ellos conocen su método y, a pesar de la dureza, suelen repetir. Entre los actores-fetiche de su cine se encuentran Timothy Spall, Alison Steadman, David Thewlis, Imelda Staunton, Ruth Sheen, Lesley Manville, Karina Fernández o Marion Bailey.

El método de Leigh se puede estructurar en dos fases. La primera dedicada a los ensayos y que incluye los pre-ensayos y la interrelación entre los actores. La segunda fase está dedicada a la estructuración del filme en la que se rueda y se edita el mismo (Clements, 1983).

La fase de los ensayos es de gran importancia en el cine de Mike Leigh y el tiempo que le dedica ha ido evolucionando a lo largo de su filmografía. Entre los ejemplos se encuentra Life is sweet, en la que los ensayos se prolongaron durante tres meses, y Another year, su último filme, en la que dicha fase se extendió durante dieciocho meses. En los filmes de Leigh, el rodaje suele extenderse a aproximadamente nueve semanas (Movshovitz, 2000: 31). 
Leigh comienza el viaje de la realización de sus filmes con una vaga idea (por ejemplo, en el caso de Vera Drake sabía que quería hacer una historia relacionada con el aborto en el Londres de los años cincuenta). El desconocimiento preciso sobre lo que va a suceder en la película, tanto por parte de Leigh como por sus actores, es un método que el director emplea para evitar ideas preconcebidas, para investigar y descubrir ideas que no surgirían si se tuviera un guión cerrado.

Después de elegir a sus actores, Leigh trabaja individualmente con cada uno de ellos y les prohíbe hablar entre ellos acerca de su propio personaje. Ningún actor posee una visión global de la narración ni es consciente de lo que está sucediendo fuera de su personaje y de sus propias escenas; desconoce las vidas de los demás tal y como sucede en la vida real (Movshovitz, 2000: 117). Por ejemplo, los actores que interpretan a Wendy y a Andy en Life is sweet, desconocían el problema de bulimia de su hija -Nicola en el filme- hasta que lo vieron en la pantalla. Este tipo de acercamiento -según Leigh- libera la creatividad del actor al no sentir la presión de obtener resultados, simplemente tiene que experimentar de un modo verdadero lo que su personaje está descubriendo en ese momento, tal y como sucede en la vida real.

Leigh suele pedir a sus actores que para su primera cita confeccionen una lista de personas que conozcan. De entre ellas, se eligen a unas tres o cuatro para construir a un personaje tridimensional similar a una persona real. Con la ayuda de Leigh, se crea un alter ego para cada actor utilizando la improvisación para averiguar el contexto, la educación, la clase social y otros detalles de los personajes, recorriendo con ellos años y años de sus vidas.

La improvisación es un método muy importante en la construcción de los filmes dirigidos por Mike Leigh, ya que supone una fuente inagotable de resultados creativos. La gran cantidad de improvisaciones que se realizan a lo largo del proceso de construcción de la película no tiene ninguna relación con lo que finalmente aparece en la pantalla, pero son importantes para que el actor se familiarice con el personaje y obtenga un resultado verosímil en su interpretación. El trabajo de Leigh en este proceso individual consiste en ir conduciendo a los actores y sus personajes en la dirección de lo que es dramático y cinemático.

La fase de pre-ensayos finaliza en el momento en que el actor conoce a la perfección y se ha acostumbrado a ser el personaje en sí mismo. Es entonces cuando está preparado para interactuar con otros personajes (otros actores) que han recibido la misma preparación (Raphael, 2008: 21).

Leigh conduce a los personajes del filme presentándolos entre ellos de modo progresivo y a través de la improvisación, mediante la que desarrollan relaciones con otros personajes que el director supervisa y dirige hacia conflictos dramáticos de algún tipo.

Las improvisaciones realizadas durante esta fase pueden prolongarse varias horas sin interrupción y, tras ellas, los actores discuten sobre su personaje (siempre en tercera persona), lo que resulta correcto o incorrecto para que tenga un sentido dramático. De nuevo, las improvisaciones no ofrecen un resultado específico sino que simplemente pueden sugerir - por ejemplo- una escena. El trabajo de Leigh consiste en destilar esas horas surgidas mediante los ejercicios de improvisación, en acciones narrativas que sucedan en unos minutos. 


\subsubsection{La estructura de un cine sin guión}

En los títulos de crédito del cine de Mike Leigh aparece devised and directed by (ideado y dirigido por), en vez de written and directed by (escrito y dirigido por). Es otro de los elementos que llaman la atención en el cine de Leigh: la inexistencia de un guión -en el sentido clásico del término-que sirva de guía para los miembros del equipo. Junto a los actores que se involucran en sus proyectos, Leigh va construyendo su universo fílmico sin necesidad de que exista un guión convencional.

Para el director británico el guión es un instrumento financiero para que los productores sepan cuánto dinero deben invertir. Su método de trabajo no incluye la discusión previa con los productores porque interfieren demasiado en el proceso creativo. Si se le pregunta cómo consigue la financiación, la respuesta es que a menudo no la consigue, aunque Simon Channing-Williams ha producido sus películas más exitosas.

A pesar de la inexistencia de un guión propiamente dicho y de la importancia de la improvisación como método de investigación durante la etapa de los ensayos, el cine de Leigh no se improvisa delante de la cámara.

Una vez finalizada la fase de ensayos, el director llega a una premisa del filme, una estructura muy breve y sencilla de tres o cuatro páginas. En el caso de Life is sweet, en dicha estructura podría leerse por ejemplo: Escena 1: Wendy en la clase de baile. Escena 2: Wendy se va a casa. Tras obtener esa sencilla estructura, el director y los actores se trasladan a las localizaciones reales en las que cada escena específica se improvisa de nuevo, se perfecciona, y cuando se obtiene el resultado esperado, se fija y se ensaya. En el momento de rodar, cada una de las escenas mencionadas está trabajada de un modo tan preciso, que no hay lugar para la improvisación.

Aunque los actores son el eje central del método de Leigh, también invita al resto del equipo de la película a embarcarse en su próximo filme. En vez de indicarles lo que deben hacer según los dictámenes del guión, se les expone cuál será el espíritu general de la película y cada uno de ellos realiza su propia investigación (Cardullo, 2010: 19).

La audiencia no es consciente de la cantidad de metraje que llega a la sala de montaje y que queda descartado. No obstante, dicho material es de gran valía para la creación y el fortalecimiento del personaje por parte del actor. Lo que la audiencia ve es simplemente la punta del iceberg creado por Leigh y por sus actores.

Entre los ejemplos de los resultados actorales del método de trabajo desarrollado por Mike Leigh destaca -en el filme Secrets and lies- la escena en la que madre e hija (a la que dio en adopción) se encuentran por primera vez. En el mismo filme, cabe destacar la escena final de la barbacoa, fruto de una improvisación de doce horas, que en el montaje final se convierte en veinte minutos (Raphael, 2008: XIX).

En Vera Drake (Vera Drake, 2004), tras una improvisación de diez horas, surge una de las mejores escenas desde el punto de vista interpretativo, en la obra de Leigh. Es el momento en el que la policía irrumpe en la casa de Vera Drake, en medio de una celebración familiar, para arrestarla. El rostro de la protagonista -que desconocía lo que iba a suceder- refleja el miedo a las consecuencias de sus actos, en ella y en su familia. La verdad presente en ese instante narrativo captado por la cámara no es fácil de lograr a través de otros métodos de dirección de actores. La actriz protagonista 
de Vera Drake -Imelda Staunton- califica su interpretación en la película como la experiencia más extraordinaria de su vida laboral, y recibe por su interpretación numerosos galardones (Raphael, 2008: XV). Entre ellos, el del festival de cine de Venecia de ese año y la nominación a mejor actriz por la Academia de cine de Hollywood.

\section{El estilo cinematográfico de Mike Leigh}

En este apartado se ofrecen los resultados obtenidos tras la realización de un análisis en profundidad de las variables más relevantes relacionadas con el estilo cinematográfico de los filmes de Mike Leigh, desde Life is sweet hasta Happy-go-Lucky. Específicamente se centra en la duración y la escala de los planos, y en la movilidad de la cámara.

\subsection{La duración del plano: los planos-secuencia como paradigma de una estética de la realidad}

En el cine de Mike Leigh, aunque la mayoría de los planos posee una duración media que se sitúa entre los 0 y los 5 segundos, si se tiene en cuenta el conjunto de los filmes analizados, la duración media del plano es de 6,58 segundos, es decir, bastante superior a la media propia del cine comercial norteamericano entre 1988-1993 (5,85 segundos) y entre 1994-1999 (4,92 segundos), y más próxima a la del cine británico entre 1988-1993 (7,4 segundos) y 1994-1999 (6,8 segundos) (Salt, 2006: 321-325). En resumen, el valor descrito se sitúa por encima de la media propia del cine comercial norteamericano de los años noventa -con cinco segundos aproximadamente- y más próxima a la del cine británico de esa misma década, de casi siete segundos.

Tal y como se muestra en la tabla 1 , Life is sweet se encuentra por encima de la media del cine británico y, por supuesto, del cine norteamericano entre 1988 y 1993; Naked y Secrets and lies poseen un valor medio ligeramente por encima del presente en el cine británico de dichas décadas; en el caso de Career girls y Topsy-Turvy se encuentra entre la media existente en el cine norteamericano y el británico entre 1994 y 1999; y la duración media de los tres últimos filmes se sitúa más próxima a la existente en el cine británico de las citadas décadas.

Tabla 1. Estadísticos descriptivos. Duración del plano (segundos).

\begin{tabular}{|l|c|c|c|c|}
\hline & N & Mínimo & Máximo & Media \\
\hline Life is sweet & 210 & 0,2 & 148 & 8,4 \\
\hline Naked & 242 & 0,1 & 125 & 7,7 \\
\hline Secrets and lies & 241 & 0,2 & 460 & 7,2 \\
\hline Career girls & 243 & 0,2 & 54 & 5,1 \\
\hline Topsy-Turvy & 268 & 0,2 & 104 & 5,3 \\
\hline All or nothing & 239 & 0,2 & 58 & 6,3 \\
\hline Vera Drake & 238 & 1 & 59 & 6,3 \\
\hline Happy-go-Lucky & 242 & 0,2 & 167 & 6,9 \\
\hline
\end{tabular}


El hecho de que todos los filmes de Mike Leigh poseen, al menos, algún plano cuya duración es como mínimo de 54 segundos es una característica que llama la atención. También están presentes algunos casos en los que la duración media del plano se sitúa muy por encima de la media como los 460 segundos de un plano presente en el filme Secrets and lies; los 167 segundos de Happy-go-Lucky; los 148 segundos de Life is sweet; o los 125 segundos de Naked. Además, la presencia de planos de una extensión muy superior a la media aumenta progresivamente según avanza la narración en cada filme. Es más, no existe una evolución hacia una menor duración de los planos en el conjunto de la filmografía analizada, es decir, no existe una reducción de la duración media de los planos en la evolución de su cine.

Los planos en los que la longitud es superior coinciden con los que poseen una mayor intensidad dramática mostrada a través de los sentimientos, emociones y diálogos de sus personajes, y no a través de la acción física propiamente dicha.

Siguiendo con el ejemplo de Secrets and lies citado en el párrafo anterior, el plano-secuencia de 460 segundos de duración coincide con el momento narrativo en el que madre e hija se encuentran por primera vez.

La existencia de planos-secuencia de larga duración, como afirma Bazin (2000), no sólo participa de una estética de la realidad, sino que implica una actitud mental más activa por parte del espectador.

\subsection{El paisaje del rostro humano: la escala del plano en el cine de Mike Leigh}

El cine de Mike Leigh, tal y como muestra la tabla 2, lejos de centrarse mayoritariamente en primerísimos primeros planos o en espectaculares planos generales de gran extensión y mayor presupuesto, emplea escalas de plano que permiten al espectador centrar su atención en el rostro de los personajes, en sus emociones y en sus diálogos. Leigh fomenta la observación detallada pero con cierta distancia, ya que la escala de plano con mayor presencia en el conjunto de sus filmes incluye, en primer lugar, el Primer Plano Medio (PPM), el Plano Medio (PM) y el Primer Plano (PP). A continuación y por orden, las escalas de plano más empleadas son el plano americano (PA), el primerísimo primer plano (PPP) y los planos que incluyen varias escalas (varios). Finalmente, las escalas menos utilizadas son el plano general largo (PGL), el plano detalle (PD) y el plano general (PG). Esta tendencia estilística del cine de Leigh es contraria a la presente en el cine comercial norteamericano que abarca las mismas décadas (Salt, 2006: 335-337).

Si nos detenemos en la evolución de la tipología de plano a lo largo del desarrollo narrativo de los filmes de Leigh, tal y como muestra la tabla 3, el PPM mantiene siempre el porcentaje más alto, es decir, en ningún cuartil le supera en porcentaje otra escala de plano. La tabla 3 también indica que la escala PD es la menos utilizada en la totalidad de los filmes y prácticamente desaparece si consideramos su evolución a lo largo de la filmografía. Por lo tanto, el detalle de ciertos elementos es menos imprescindible en los filmes que se sitúan hacia el final de su obra. 
Tabla 2. Resultados descriptivos. Escala de plano.

\begin{tabular}{|l|c|c|}
\hline & Frecuencia & Porcentaje \\
\hline PD & 38 & $2,0 \%$ \\
\hline PPP & 183 & $9,5 \%$ \\
\hline PP & 348 & $18,0 \%$ \\
\hline PPM & 567 & $29,3 \%$ \\
\hline PM & 364 & $18,9 \%$ \\
\hline PA & 166 & $8,6 \%$ \\
\hline PG & 47 & $2,4 \%$ \\
\hline PGL & 58 & $3,0 \%$ \\
\hline Varios & 152 & $7,9 \%$ \\
\hline Total & 1923 & $99,4 \%$ \\
\hline
\end{tabular}

En la totalidad de películas analizadas, el máximo porcentaje de la escala PPM se concentra en el $4^{\circ}$ cuartil, es decir hacia el final del desarrollo narrativo. En el caso de la segunda tipología más utilizada (PM), el máximo porcentaje se sitúa en el primer cuartil; y en el segundo cuartil, en el caso de la tercera tipología más aplicada (PP). Por lo tanto, en el cine de Mike Leigh la cantidad de planos más cortos no aumenta hacia el final del desarrollo narrativo.

Tabla 3. Tabla de contingencia. Evolución de la escala de plano.

\begin{tabular}{|l|c|c|c|c|}
\hline & $\begin{array}{c}\mathbf{1}^{\mathbf{0}} \\
\text { cuartil }\end{array}$ & $\begin{array}{c}\mathbf{2}^{\mathbf{0}} \\
\text { cuartil }\end{array}$ & $\begin{array}{c}\mathbf{3}^{\mathbf{0}} \\
\text { cuartil }\end{array}$ & $\begin{array}{c}\mathbf{4}^{\mathbf{0}} \\
\text { cuartil }\end{array}$ \\
\hline PD & $2,3 \%$ & $1,6 \%$ & $2,9 \%$ & $0,9 \%$ \\
\hline PPP & $3,5 \%$ & $5,6 \%$ & $15,3 \%$ & $13,8 \%$ \\
\hline PP & $15,5 \%$ & $23,0 \%$ & $18,3 \%$ & $16,0 \%$ \\
\hline PPM & $30,7 \%$ & $30,6 \%$ & $25,9 \%$ & $31,0 \%$ \\
\hline PM & $21,7 \%$ & $18,2 \%$ & $19,1 \%$ & $16,3 \%$ \\
\hline PA & $10,2 \%$ & $7,9 \%$ & $6,9 \%$ & $9,6 \%$ \\
\hline PG & $2,5 \%$ & $2,5 \%$ & $2,2 \%$ & $2,7 \%$ \\
\hline PGL & $2,5 \%$ & $3,6 \%$ & $3,1 \%$ & $2,9 \%$ \\
\hline Varios & $11,1 \%$ & $7,0 \%$ & $6,3 \%$ & $6,9 \%$ \\
\hline Total & $\mathbf{5 2 1}$ & $\mathbf{4 4 4}$ & $\mathbf{5 0 9}$ & $\mathbf{4 4 9}$ \\
\hline
\end{tabular}

\subsection{La quietud de la cámara en el cine de Leigh}

El universo fílmico creado por Mike Leigh se caracteriza por la preeminencia del estatismo de la cámara, tal y como muestra la tabla 4. En esa misma tabla se observa que, de toda la filmografía, Secrets and lies y All or nothing son los filmes más estáticos. Career girls, por el contrario, es el más dinámico con un valor muy por encima de la media de las restantes obras, aunque la movilidad alcanza un escaso $30 \%$. 
Tabla 4. Frecuencias de estatismo/dinamismo.

\begin{tabular}{|l|c|c|}
\hline & Estático & Dinámico \\
\hline Life is sweet & $84,8 \%$ & $15,2 \%$ \\
\hline Naked & $88,0 \%$ & $12,0 \%$ \\
\hline Secrets and lies & $92,9 \%$ & $7,1 \%$ \\
\hline Career girls & $69,5 \%$ & $30,5 \%$ \\
\hline Topsy-Turvy & $89,2 \%$ & $10,8 \%$ \\
\hline All or nothing & $91,2 \%$ & $8,8 \%$ \\
\hline Vera Drake & $86,1 \%$ & $13,9 \%$ \\
\hline Happy-go-Lucky & $85,5 \%$ & $14,5 \%$ \\
\hline
\end{tabular}

Tal y como se muestra en la tabla 5, entre los escasos movimientos de cámara presentes en la obra de Mike Leigh, destaca la panorámica horizontal (7,9\%); la vertical $(5,2 \%)$; la cámara al hombro $(4,2 \%)$; el desplazamiento de la cámara en soporte estático (track) (2,0\%); el desplazamiento de la cámara hacia delante $(1,2 \%)$; el desplazamiento de la cámara hacia atrás $(1,1 \%)$; el desplazamiento lateral de la cámara $(0,9 \%)$; el desplazamiento de la cámara hacia arriba $(0,2 \%)$; y el desplazamiento de la cámara hacia abajo $(0,1 \%)$. De entre los movimientos citados, están presentes en mayor o menor medida en la mayoría de los filmes, la panorámica horizontal y la vertical, el desplazamiento de la cámara en soporte estático (track), el desplazamiento de la cámara hacia delante, hacia atrás y lateral.

Tabla 5. Frecuencias de los movimientos de cámara.

\begin{tabular}{|c|c|c|c|c|c|c|c|c|c|}
\hline & Life & Naked & Secrets & Career & Topsy & All or & Vera & Happy & Media \\
\hline Hombro & $0,0 \%$ & $2,5 \%$ & $1,7 \%$ & $25,5 \%$ & $0,4 \%$ & $1,7 \%$ & $0,0 \%$ & $1,2 \%$ & $4,2 \%$ \\
\hline Track & $1,9 \%$ & $1,7 \%$ & $0,4 \%$ & $0,4 \%$ & $1,9 \%$ & $1,7 \%$ & $3,4 \%$ & $10,0 \%$ & $2,7 \%$ \\
\hline Lateral & $0,5 \%$ & $0,4 \%$ & $0,4 \%$ & $0,4 \%$ & $1,1 \%$ & $0,8 \%$ & $0,4 \%$ & $3,3 \%$ & $0,9 \%$ \\
\hline $\begin{array}{c}\text { Pan. } \\
\text { horizontal }\end{array}$ & $10,0 \%$ & $14,0 \%$ & $5,8 \%$ & $3,3 \%$ & $7,5 \%$ & $4,2 \%$ & $8,4 \%$ & $9,9 \%$ & $7,9 \%$ \\
\hline $\begin{array}{c}\text { Pan. } \\
\text { vertical }\end{array}$ & $6,2 \%$ & $7,9 \%$ & $1,2 \%$ & $3,3 \%$ & $7,1 \%$ & $2,1 \%$ & $8,0 \%$ & $5,8 \%$ & $5,2 \%$ \\
\hline $\begin{array}{c}\text { Hacia } \\
\text { arriba }\end{array}$ & $0,0 \%$ & $0,0 \%$ & $0,0 \%$ & $0,0 \%$ & $0,0 \%$ & $0,0 \%$ & $0,0 \%$ & $0,8 \%$ & $0,1 \%$ \\
\hline $\begin{array}{c}\text { Hacia } \\
\text { abajo }\end{array}$ & $0,0 \%$ & $0,0 \%$ & $0,0 \%$ & $0,4 \%$ & $0,4 \%$ & $0,0 \%$ & $0,0 \%$ & $0,0 \%$ & $0,1 \%$ \\
\hline $\begin{array}{c}\text { Hacia } \\
\text { delante }\end{array}$ & $0,5 \%$ & $0,8 \%$ & $0,4 \%$ & $2,9 \%$ & $0,4 \%$ & $0,8 \%$ & $2,1 \%$ & $1,7 \%$ & $1,2 \%$ \\
\hline $\begin{array}{c}\text { Hacia } \\
\text { atrás }\end{array}$ & $1,0 \%$ & $0,8 \%$ & $0,8 \%$ & $3,3 \%$ & $1,1 \%$ & $0,4 \%$ & $0,0 \%$ & $1,2 \%$ & $1,1 \%$ \\
\hline \begin{tabular}{c} 
Circular \\
\hline
\end{tabular} & $0,0 \%$ & $0,0 \%$ & $0,0 \%$ & $0,0 \%$ & $0,0 \%$ & $0,0 \%$ & $0,4 \%$ & $0,0 \%$ & $0,1 \%$ \\
\hline
\end{tabular}


Por lo tanto, la filosofía de Leigh se inclina a desplazar la cámara en momentos muy concretos de sus narraciones, como por ejemplo, la cámara al hombro para mostrar la violencia de la escena inicial del filme Naked, o para trasladarnos al pasado en las escenas de flashback de Career girls.

Aunque la totalidad de la filmografía de Mike Leigh es eminentemente estática, en la tabla 5 se detecta la introducción de ciertos movimientos de mayor complejidad, sobre todo a partir de Career girls, que es un filme situado hacia la mitad de su filmografía. Entre ellos, la traslación de la cámara en soporte estático (track), posee en Vera Drake y en Happy-go-Lucky, un porcentaje muy reducido aunque ligeramente superior al resto de películas; el desplazamiento de la cámara hacia delante se encuentra ligeramente por encima de la media en Career girls y en Vera Drake; el movimiento de la cámara hacia arriba sólo está presente en Tospy-Turvy y en Happygo-Lucky; y el desplazamiento de la cámara hacia abajo sólo está presente en Career girls y en Tospy-Turvy.

Leigh emplea mayoritariamente movimientos de cámara sencillos y anclados a la tierra, evitando los movimientos aéreos, los circulares o los realizados con sofisticadas grúas.

La práctica ausencia de movimiento en el cine del director británico llama la atención en un contexto fílmico internacional en el que la tendencia es hacia el incremento de la movilidad de la cámara. De hecho, tal y como muestra la tabla 6, en la mayoría de los filmes de Mike Leigh se produce un incremento del estatismo de la cámara según avanza la narración, es decir, hacia el final del desarrollo narrativo coincidiendo con el momento climático. Esta característica convierte el estilo de Mike Leigh en contrario a la tendencia imperante desde finales de los ochenta y sobre todo, a partir de los noventa, en el cine comercial norteamericano, que enfatiza la movilidad de la cámara hacia el final del desarrollo narrativo coincidiendo con el momento climático (Salt, 2006: 338-339).

Tabla 6. Tabla de contingencia.

\begin{tabular}{|l|c|c|c|c|}
\hline & $\mathbf{1}^{\mathbf{0}}$ Cuartil & $\mathbf{2}^{\mathbf{0}}$ Cuartil & $\mathbf{3}^{\mathbf{0}}$ Cuartil & $\mathbf{4}^{\mathbf{0}}$ Cuartil \\
\hline Estático & $81,8 \%$ & $85,6 \%$ & $87,8 \%$ & $89,1 \%$ \\
\hline Dinámico & $18,2 \%$ & $14,4 \%$ & $12,2 \%$ & $10,9 \%$ \\
\hline
\end{tabular}

\section{Conclusiones}

Entre las principales conclusiones halladas en esta investigación se encuentra que Mike Leigh es un director de cine comprometido con la búsqueda y la creación de verdad a través de todas las fases que conforman su cine. La filosofía del autor unifica los dos conceptos fundamentales de su cine: la hibridación entre lo real y el artificio, entre lo documental y la ficción, que condiciona la totalidad de su obra, desde la idea original hasta su realización, desde el método de trabajo hasta su estilo cinematográfico.

Entre los presupuestos éticos de Leigh se encuentra una clara voluntad por diferenciarse del cine comercial -especialmente el norteamericano- en el que no 
encuentra ninguna verdad. De ahí que, desde sus inicios como creador, busca a través de la experimentación y de la vanguardia, técnicas que definen su posterior método de trabajo. El autor confiere una gran importancia a la improvisación con los actores como método de investigación de la vida real que conduce al descubrimiento del filme que quiere realizar. Este hecho demuestra la importancia del hallazgo en el cine de Mike Leigh, método para obtener ideas novedosas y eliminar las preconcebidas sobre lo que puede suceder en un filme, como las presentes en un guión clásico con su planteamiento, nudo y desenlace.

Leigh encuentra verdad en un tipo de cine en el que destaca el concepto de colaboración o autoría colectiva, un modo cooperativo de trabajar en el que -lejos de erigirse como autor total- pretende que todos los miembros del equipo fílmico -especialmente los actores- aporten con su trabajo creativo a la obra final. El actor es el eje central y motor de la creación fílmica de Mike Leigh, condiciona toda su obra y los quiere reales, tridimensionales, alejados de los personajes estereotipados, interpretados por actores-estrella presentes en el cine comercial.

El compromiso con la búsqueda de la verdad de Leigh se pone de manifiesto a través de determinadas elecciones en lo referente a los elementos más relevantes del estilo cinematográfico, claramente contrapuestos a los predominantes en el cine comercial norteamericano, caracterizado por la preeminencia de los primerísimos primeros planos, de duración cada vez más corta y en el que proliferan los movimientos de cámara complejos. Mike Leigh, centrado en la búsqueda de la verdad del ser humano, decide emplear escalas de plano centradas en la emoción del rostro y en el diálogo de los actores. Dicha verdad también está presente a través del respeto por el tempo del actor y de la narración que se muestra a través de una duración media del plano superior a la del cine comercial norteamericano. La presencia en su cine de planos-secuencia de larga duración muestra la importancia que el director confiere a determinados momentos narrativos, permitiendo que la acción se desarrolle dentro del cuadro y que el espectador realice su propio découpage.

Finalmente, la búsqueda de la verdad del director y su falta de interés por la espectacularidad de los resultados visuales se muestra a través de una cámara preeminentemente estática, que sólo se mueve en momentos narrativos muy concretos. Esta característica de una cámara que no dicta hacia dónde dirigir la mirada ni promueve la participación física en la acción obliga a que el espectador se esfuerce sobre el material visionado. Esto último indica que la filosofía de Mike Leigh también se pone de manifiesto a través del nivel de exigencia hacia el espectador, contraria a la presente en el cine comercial contemporáneo. La búsqueda de la verdad del cine del director británico requiere del esfuerzo de una audiencia a la que se le plantean más preguntas que respuestas y a la que se deja libre para que se forme su propia opinión sobre los personajes y sus acciones. 


\section{Referencias bibliográficas}

BAZIN, André (2000). Qué es el cine. Madrid: Rialp.

BORDWELL, David (2006). The way Hollywood tells it. Berkeley and London: University of California Press.

CARDULLO, Bert (2010). Loach and Leigh, Ltd.: The Cinema of Social Conscience. Cambridge: Cambridge Scholars Publishing.

CARNEY, Ray (2000). The films of Mike Leigh. Embracing the world. Cambridge: Cambridge University Press.

CLEMENTS, Paul (1983). The improvised play: The work of Mike Leigh. London: Methuen.

CORTÉS-SELVA, Laura y CARMONA-MARTÍNEZ, Mercedes (2014). Propuesta metodológica para el análisis del estilo visual de los filmes: un acercamiento empírico. En: UBIERNA, Francisco; SIERRA, Javier (coord.). Miscelánea sobre el entorno audiovisual en 2014. Madrid: Fragua.

-(2013). El sello del estilo visual de Dick Pope y Mike Leigh: análisis de su obra cinematográfica. En: Historia y Comunicación Social, vol. 18, N Esp. Octubre, pp. 491-501.

COVENEY, Michael (1996). The world according to Mike Leigh. London: Harper-Collins.

IRVINE, Ian K.(2008). But what I really want to do is write : adapting the Mike Leigh Method for writers for the stage. Masters by Research thesis: Queensland University of Technology.

LEIGH, Mike (2002). All or nothing. London: Faber and Faber.

-(1999). Topsy-Turvy. London: Faber and Faber.

- (1997a). Secrets and lies. London: Faber and Faber.

-(1997b). Career girls. London: Faber and Faber.

-(1995). Naked and other screenplays. London: Faber and Faber.

MOVSHOVITZ, Howie (ed.) (2000). Mike Leigh interviews. Jackson: University Press of Mississippi.

O’SULLIVAN, Sean (2011). Mike Leigh. Urbana: University of Illinois Press.

RAPHAEL, Amy (ed.) (2008). Mike Leigh on Mike Leigh. New York: Faber \& Faber. SALT, Barry (2006). Moving into pictures. London: Starword.

WATSON, Garry (2004). The cinema of Mike Leigh: A sense of the real. London: Wallflower press.

WHITEHEAD, Tony (2007). Mike Leigh. Manchester: Manchester University Press. 